\title{
Integrating the Two Singing Methods of Folk Style Singing and Bel Canto in Teaching
}

\author{
Zhang Zhaomeng \\ Sichuan University of Culture and Arts, Sichuan, Mianyang, 621000
}

Keywords: folk style singing method; bel canto; fusion

\begin{abstract}
In today's emphasis on core literacy and quality education, education not only bears the important responsibility of imparting scientific and cultural knowledge to students, but also emphasizes the cultivation of students' comprehensive qualities and abilities, and more reflects the role of music teaching. Vocal music is the key content of music teaching. The two kinds of singing methods of the folk style and bel canto are two kinds of singing forms of vocal music teaching. There are both similarities and differences between the two. Therefore, the analysis of the fusion of national and melody in vocal music teaching is particularly important and necessary.
\end{abstract}

\section{Introduction}

With the continuous development of economic globalization, trade and economic cooperation have gradually increased among countries, and cultural exchanges have become more frequent. In the teaching of vocal music, incorporating the vocal singing method in the folk style singing method, promoting and supporting the improvement and development of the national vocal music, can not only improve the national vocal music, but also make the national vocal music learn from each other and learn from each other. Integration, while effectively stimulating students' enthusiasm for learning, improving the quality and efficiency of classroom teaching, can also greatly enhance the comprehensive ability of students' vocal singing and improve their artistic accomplishment.

\section{The origin of the two singing methods of folk style singing and bel canto}

\subsection{Bel canto}

Bel Canto is a method of singing or vocalization formed by western countries. This singing method originated from Italy. The word "beautiful singing" is the meaning of wonderful singing. In the hearts of the people of all countries including Italy, they feel that the beautiful singing method reflects strict skills and standards, pointing out that this is a singing method of scientific norms. Due to the scientific nature of this singing method, some national singers continue to learn from and cite the singing techniques and vocal methods of the vocal singing in the process of interpreting their national songs and artistic songs, reflecting the different styles of songs and reflecting the remarkable features of the country. National characteristics. The fusion of different singing methods led to the birth of different vocal music schools in various countries, such as the French School, the German School, and the Russian School. Bel Canto pays more attention to the formalization and standardization of singing. For singers and vocals, there is a set of clear requirements. Any step reflects different characteristics. In terms of results and form, its operability is particularly strong.

\subsection{Folk style singing}

The folk style singing method is an aesthetic principle and traditional custom formed by the long-term development and evolution of all ethnic groups in China. It fully reflects the singing characteristics and musical image of the Chinese national melody, the tone of the voice, the spiritual features, and the personality characteristics, to interpret different regions and different regions of China. The folk style singing method of the vocal music works of different nationalities. The folk style singing method is directly related to the local folk customs, local dialects, and national 
languages. It is based on the national language, based on the national culture, with the charm and the line as the advantage, and the words, the cavity, the emotion and the sound complement each other. The art of singing. During its development, folk art slowly established an art characterized by "songs and dances, combination of singing and vocalization, both form and spirit, and the words are in perfect harmony." The folk style singing method does not put forward special requirements for singers. It has not yet established scientific and systematic training materials, vocal methods and training modes. It pays more attention to the singers' cheerful dance, bright costumes and sweet sounds. The ancient art that has been passed down from generation to generation reflects stronger popularity and folk character.

\section{The difference between the two singing methods of folk style singing and bel canto}

\subsection{The vocal singing method includes different singing methods, and the folk style does not distinguish the singing methods}

Bel Canto is divided into high and low singing, so it distinguishes the singer's voice in detail: male voice includes bass, baritone, tenor, female voice including mid-tone, soprano. For the characteristics of treble, the tenor includes lyrical tenor, hero tenor and dramatic tenor. There are also many singers in the bass and baritone. The soprano usually includes a lyrical soprano, a flower soprano and a dramatic soprano. Operas that choose Bel Canto usually attach great importance to singing skills. Their stage singing style maintains a similar style in any part of the world, reflecting a high standard and normative. It emphasizes the fullness, grace and sweetness of the singer's voice. When singing, the singer reveals rich emotions, even if the emotions that are unhappy, are expressed in a beautiful way. Bel Canto requires the singer to have a strong ability to use the voice. Because the singing requirements are particularly strict, the sound zone is very uniform, the mixing sound is particularly in place, and the sound range is very broad. The most striking feature of the Bel Canto is its individualized distinction between high school and low voice.

\subsection{When the Bel Canto sings, it is necessary to carry out a breath confrontation, and the folk style singing method does not emphasize}

The vocal vocalization should reflect strong resonance and overtones. The bass should be strong, the treble should be transparent, the sound should be full, the sound quality should be metallic, and the tone should be pure. Because the vocalization emphasizes "erecting" and "concentration", the sounds of the various parts can be properly blended to spread a farther distance with a thicker tone. The singer's free use of breath can bring a feeling of incompetence to the audience. The folk singing method is to interpret the special charm of a specific place, a specific group, and a nationality by means of its singing voice. The singing methods of the same people show relatively uniform norms within the nation. Of course, there are exceptions. For example, some local folk minors have different styles and singing methods, and there are different forms of folk singing. All in all, those who speak loudly, have a thin voice, and are small in size are suitable for applying folk singing. Their voice is the original singing method. After systematic training, they can sing folk songs as long as they imitate.

\section{The integration of ethnic and bel canto in teaching}

In the teaching of vocal music, the two singing methods of nationality and melody can be integrated in the aspects of breathing, vocalization techniques, biting words, etc, which can improve the singing level and enhance the teaching quality at the same time.

\subsection{The combination of the two singing methods in breathing}

In terms of controlling breathing, China's national singing method has always been a self-contained system. Some people are usually in a state of tension in singing. They pay attention to the pronunciation of the lyrics but do not care about controlling the sound. Some over-emphasize the vibration of the vocal cords and throat, resulting in a monotonous, pale singing effect. However, 
in bel canto singing, more attention is paid to the fusion of the cavity body, especially to the control of the breath, and the control of the singing voice on the premise of breath, so that the words of the lyrics are not clear.Nowadays, under the premise of knowing one's own problems, China's national singing method strives to learn from the advantages of Bel Canto and promote its own development.In addition, the folk singing method has always maintained its own advantages and characteristics. By referring to the techniques of bel canto in controlling respiration, it has effectively grasped the unified and harmonious ways of breathing and pronouncing words, made up for the deficiency of the previous singing in the breath, and improved the richness and permeability of the voice.

\subsection{The combination of the two singing methods in the vocal skills}

In the past, the national singing method of our country generally paid special attention to practicing language, and proposed to enhance the training and vocalization effect through language training. Therefore, excessive attention to the expression and interpretation of the lyrics will greatly reduce the appreciation of the song, especially when using difficult singing skills. At present, the national singing method uses the advantages of the bel canto in the vocalization, and eliminates or reduces the problems in the pronunciation technique through the scientific breathing method and the means of dealing with the true false sound. As we all know, Bel Canto attaches importance to singing in a free and relaxed state. Compared with national singing, this singing technique can effectively reduce the stimulation and effect of vocalization on the throat and vocal cords, so as to more effectively bite the pronunciation and reduce the burden on the vocal organs. The integration and reference of the two singing methods of Chinese national and Western American sound can make the pronunciation of folk singing more free and easy, and enhance the effect of singing and appreciate the value. In addition, the national singing method still retains its unique singing and pronunciation advantages, further enhancing its aesthetic, appealing and expressive force.

\subsection{The combination of the two singing methods on the gnashing of characters}

Traditional folk singing emphasizes the expression of emotions and sounds, paying special attention to the clarity of the words, and requires the singer to clearly issue all the words. Therefore, the singer can only use the technique of vocalization of the front of the mouth to improve the pronunciation accuracy when singing, and the Chinese pronunciation features are reflected through the lips, but excessive use of oral pronunciation can reduce the resonance of the chest, which will reduce the thickness and fluency of the pronunciation, causing the sound volume to drop and the lack of penetration. The unique pronunciation of Italian makes the Bel Canto lower the requirements for the vocalist's pronunciation and biting. Most singers use the squeaky words to open the large joints to the fullest, making the pronunciation more round and smooth. Therefore, the national singing method in teaching has gained enlightenment from the pronunciation method of the Bel Canto, learning the pronunciation technique of the pronunciation, and on the basis of maintaining its own characteristics, the traditional horizontal spit mode is changed to the horizontal and vertical mode, thereby improving the roundness and coherence of the pronunciation and perfecting its own drawbacks.

\section{Conclusion}

After the 20th century, China gradually introduced the Bel Canto method, which had a great impact on the music field and traditional folk singing in China, and effectively promoted the development and progress of the vocal music field. In the process of continuous development of the two singing methods of folk singing and Bel Canto, the two have a tendency to influence each other and integrate with each other. The integration of the Bel Canto singing method into the national singing method can highlight the unique artistic charm. We must deeply analyze the two concepts and differences of national and Bel Canto, as well as the ways in which the two methods can be integrated, in order to better promote the development of vocal music teaching and vocal music art. 


\section{References}

[1] Cai Fengdan. Study on the aestheticism of national singing in vocal music teaching[J]. Journal of Jilin Provincial Institute of Education, 2018, 34(05): 105-107.

[2] Gao She. Study on the fusion of national singing and beautiful singing in vocal music teaching[J]. Knowledge Economy, 2018(05):113 -114.

[3] Liu Ping. The mutual reference and integration of Chinese and American singing and national singing in vocal music teaching[J]. Comparative Study of Cultural Innovation, 2018, 2(07): 189-190.

[4] Wanghui. Explore the teaching reform of national vocal music in colleges and universities from the nationalization of vocal singing[J]. Voice of the Yellow River, 2017(24): 41-42.

[5] Li Xingji. National vocal music teaching draws lessons from and integrates the beautiful singing method[J]. Art Evaluation, 2017(20): 101-102. 